\title{
Expert clinical consulting for early drug development programs in Korea
}

\author{
Hanlim Moon* \\ CUREnCARE Research, Seoul 07345, Korea \\ *Correspondence: H. Moon; Tel: +82-2-783-9249, E-mail: hanlim.moon@curencareresearch.com
}

Check for updates

pISSN: 2289-0882

elSSN: 2383-5427

\section{Introduction}

The global pharmaceutical industry has been facing a crisis over the last two decades, characterized by skyrocketing development costs, high failure rates, and dwindling returns.[1] In parallel, tectonic shifts in Korea's healthcare landscape have taken place with hundreds of newly-formed biotech companies boldly launching innovative drug discovery programs. Despite the global turmoil, this has been a very positive sign for the future of drug development in Korea. The key factors for success in early clinical development include strategic planning for program development, robust clinical development plans and sound project management. Professional staff members with specific expertise and competencies are critical for biotech companies, with in-depth knowledge, real-world experience, team work, and seamless project management all required for the achievement of success.

A diverse range of professionals are needed to facilitate the entire spectrum of the drug development process (Fig. 1).[2] Of these, project development leaders play a key role by maintaining the team structure and keeping the development strategy and execution plan on track, while being responsible for overall direction and priority decision-making. However, it is often a challenge for small pharmaceutical and biotech companies to recruit the functional members required for in-house positions, and it can sometimes seem virtually impossible to attract experienced drug development leaders to new biotech start-ups. More importantly, due to the limited experience present in such new companies, the CEOs themselves can often be unaware of the types of resources they need in the first place. Human

Copyright (C) 2019 Hanlim Moon

(a) It is identical to the Creative Commons Attribution Non-Commercial License (http://creativecommons.org/licenses/by-nc/3.0/).

(®) This paper meets the requirement of KS X ISO 9706, ISO 9706-1994 and ANSI/NISO Z.39.48-1992 (Permanence of Paper).

Reviewer

This article was invited and reviewed by the editors of TCP. resources issues can be addressed by outsourcing to specialized service providers including professional contract research organizations (CROs) when resources permit. However, this is not always a robust solution for ensuring an effective clinical development strategy, as the primary role of a CRO is to execute tasks at an operational level. To address such issues more broadly, the use of external clinical development specialists for strategic planning is needed.

Based on the results of a survey conducted by KoNECT in 2017, many of Korea's drug development companies understand the need for clinical development specialists and close consultation with CROs. However, they often receive only limited support at the early development stages rather than that required for full clinical development. In addition, many have voiced difficulties in understanding the complexity of consulting feedback, due to a lack of familiarity with the clinical development process.[3] Therefore, a supportive clinical consulting program to fill this gap is critically needed.

\section{What makes a consulltant qualified for clinical development?}

For clinical development consultants seeking to provide tailored recommendations and subsequent support, the initial qualification criteria to be considered include: 1) clinical scientists, 2) experience in the pharmaceutical industry $>10$ years, 3) experience as a clinical development specialist, 4) familiarity with product development strategy, and 5) proficiency in project management. Most importantly, prior experience as a consultant is essential, as this ensures a more wholesome understanding of how to provide the right advice to companies in different settings and with unique challenges. The provision of customized solutions based on precise analysis of lead candidate data and the competitive landscape at each stage of development is key. A wide range of specialists are needed to ensure comprehensive strategic planning, and a lead consultant should be responsible for composing the panel based on the profile 


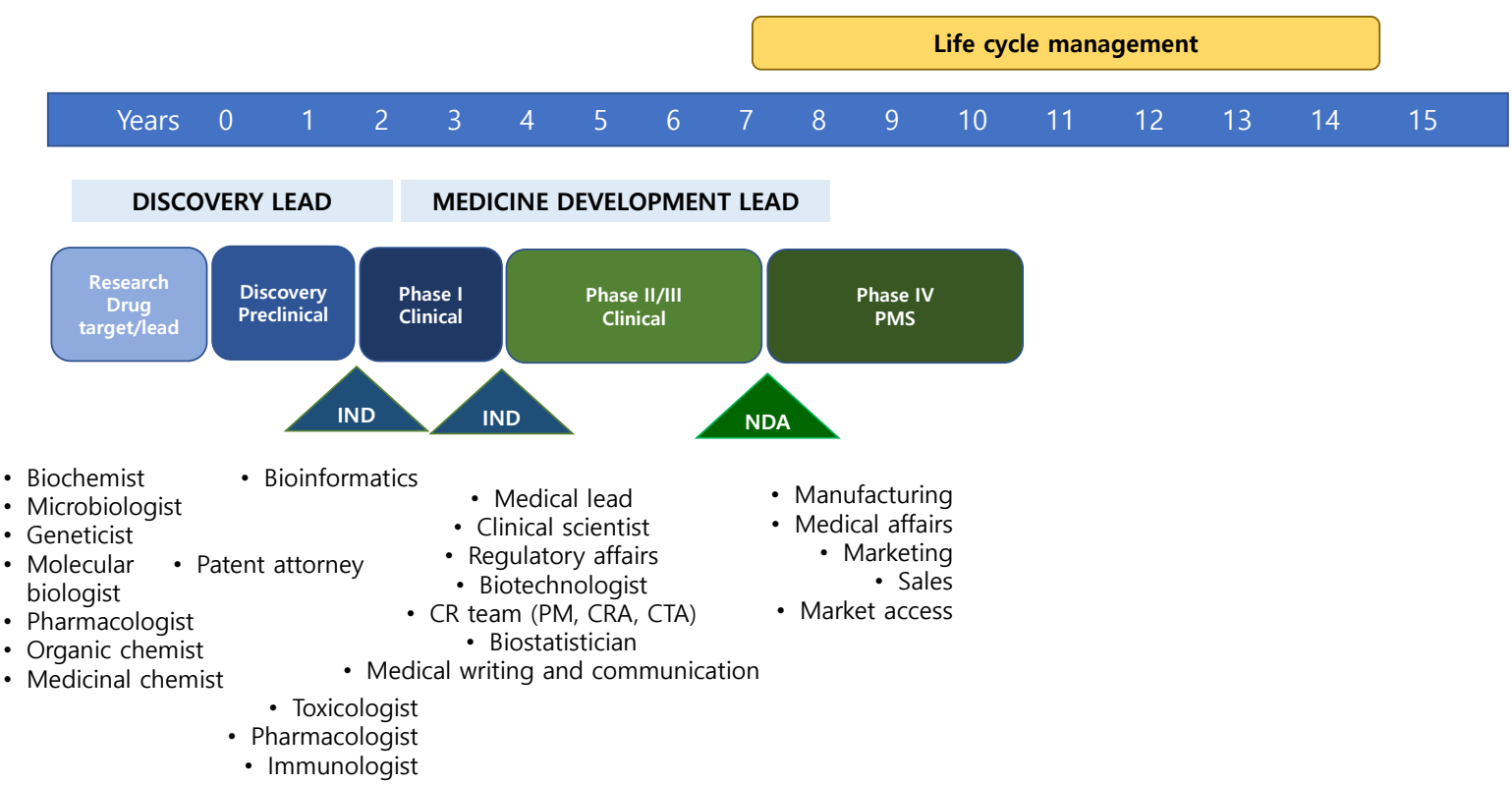

Figure 1. Specialists required throughout the drug development process.

and development status of the product. The basic panel should include, but not be limited to, clinical development specialists, therapeutic area clinicians, regulatory specialists, clinical pharmacologists, ADME specialists, CMC specialists, and biomarker specialists. Panels should have proficient experience in clinical development and extensive knowledge of the drug development process.

Consultants generally provide recommendations to client companies that are limited in scope according to the questions they receive, as is often the case for service providers. However, more effective consulting can be achieved through a proactive approach with a sense of responsibility for the project's success and understanding the limitations of their client's background knowledge. It is also helpful to create expert panels to overcome hurdles and more broadly understand the potential options available. It is not uncommon for companies to be fully dependent on clinical investigators to create clinical development plans and other trial-related documents. Clinicians are essential for accurate clinical advice but cannot be expected to play the role of a drug development leader, unless they have specific experience themselves. For this reason, when drug development decisions need to be made, they may not be the most suitable party to turn to. If clinicians aspire to create early proof of concept data through investigator-initiated trials, they can become excellent contributors. However, if companies only require clinicians for clinical development, the resultant scope should be limited to their specific expertise.

\section{Timing and scope of clinical development consulting}

It is ideal to begin consultations with clinical development specialists when non-clinical studies are underway or earlier. Clinical development plans should be based on the mechanism of action, relying on data from non-clinical efficacy and safety studies. It is common to see projects needing to return to the non-clinical stage after reaching clinical studies, due to a lack of relevant data. Gap analyses for non-clinical data should serve as the starting point for clinical development plans, with the intended indication and the expected new drug application (NDA) in mind. If gaps relating to the mechanism of action and/or non-clinical data are found when clinical trials are due to start, the entire development timeline can be compromised.

When drug developers are lacking the required expertise, they often seek consulting input limited to the clinical trial design and related items such as inclusion/exclusion criteria, and sample size calculation. However, for successful clinical development, the points to consider are considerably wider in scope than the clinical trial plan. Items for consultation include analysis of the full range of unmet medical needs, and the development scope based on mechanisms of action, the clinical development plan, IND strategy, regulatory communication, territorial decisions, development of the investigators' brochure, creation and updating of the target product profile, development of companion diagnostics, clinical trial design, outsourcing strategies and vendor management, selection of investiga- 
tors, scientific advisory board management and publication strategy.

\section{How to effectively use clinical consulting}

Drug developers need to plan the timing of consultation ideally 12 to 18 months prior to IND submission. It is recommendable to discuss with the consultant beforehand about the consulting scope, rather than simply following a list of items in their mind, as they may need more information than they realize. Companies are also sometimes reluctant to provide all the relevant documents and materials to consultants due to confidentiality concerns. It is desirable to achieve mutual trust so that all data and information necessary can be shared for analysis and decision points. Both parties should put in their best efforts to align with each other for consulting items and overall direction through continuous communication. Consulting reports should include data analysis, an understanding of the current market environment and competition, scientific and logical rationale for decision points, and finally, clear and actionable recommendations. Once the report and recommendations arrive, sponsors should read and understand them carefully, and follow up with further discussion on any uncertain points to avoid misunderstanding and to ensure the correct actions are taken. If some recommendations are declined or impractical, they should document clearly why such recommendations were not taken. For those recommendations that are accepted, structured action plans are to be set immediately with a clear timeline. If reasons for any particular decision are found to be unclear a few years later due to poor documentation, it may negatively impact the program. Logical decision-making processes and compre- hensive documentation can facilitate opportunities to correct decisions in the future, even though such remedies may be late. After the consultation is over, sponsors should convey feedback to the consultant in relation to the process and outcomes.

\section{Consulting for operational management of clinical trials}

Consulting needs for drug developers can include operational management plans for clinical studies. Clinical trial performance is highly dependent on the level of excellence in overall project and operational management. An inadequate understanding of clinical study-related tasks and milestones can result in timelines being unrealistic and unachievable. Many biotech companies choose not to use in-house resources to manage clinical trials, preferring to outsource for reasons of efficiency. To assess CROs adequately and select the right one is the first step; but managing a CRO can be another task altogether. The CRO should be hired at least 8 months before IND submission, if not earlier, and the selection process with clear assessment procedures should be conducted at least 3 months prior to this. Companies need a sponsor-side clinical project manager to oversee all activities performed by the CRO or hire a separate project manager from an independent party.

\section{Good example of an effective consulting pro- gram for clinical development support by KDDF and KONECT}

Since 2017, the Korea Drug Development Fund (KDDF) and Korea National Enterprise for Clinical Trials (KoNECT) have run a clinical consulting program called Advanced Clinical

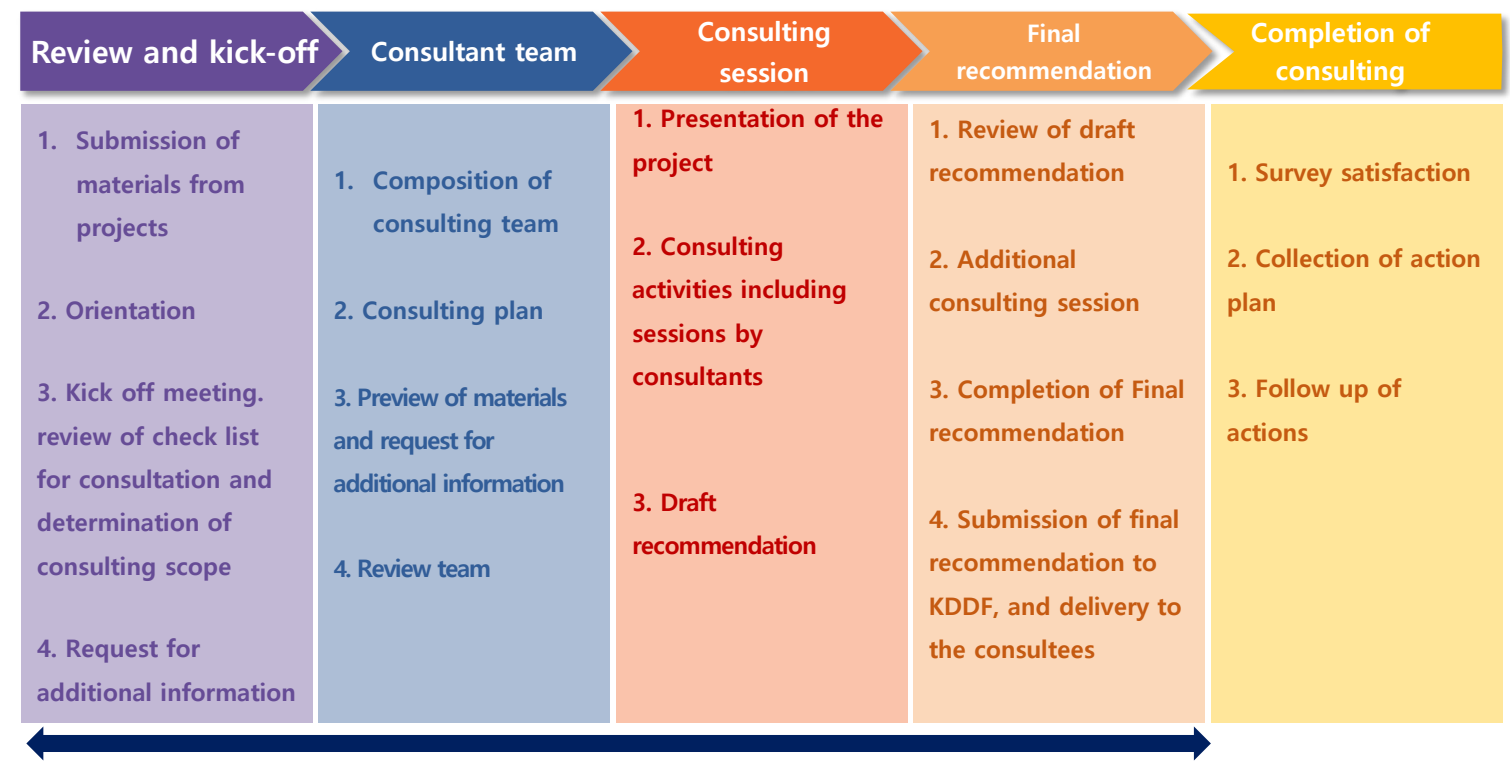

\section{5-3 months}

Figure 2. Consulting process and timeline for the ACT program (courtesy of KoNECT). 
Trials (ACT) to support small to mid-sized drug development companies who lack the internal resources and capabilities to ensure successful clinical development. It is a short-term consulting program designed to provide recommendations specific to their stage of drug development over 3 months (Fig. 2). Six projects from six different companies have benefited from the program and received effective and tailored consulting advice. However, as the support is limited to short-term consulting, the companies stand to benefit most by seeking additional advice where needed.

\section{Conclusion}

Success in clinical development requires an effective strategy, structured planning and proficient project management capabilities. The ACT program run by KDDF and KoNECT to support small pharmaceutical and biotech companies has been widely regarded as effective in delivering comprehensive consulting to those in need. For drug developers lacking the necessary resources, professional consulting is an important enabler to not only fill human resources gaps, but also to ensure customized strategies for the sponsor, a robust clinical development plan, and competent project management.

\section{Acknowledgments}

The author thanks Dr. Deborah Chee and KoNECT for their kind permission to share information for publication.

\section{Conflict of interest}

- Authors: Nothing to declare

- Reviewers: Nothing to declare

- Editors: Nothing to declare

\section{References}

1. Paul SM, Mytelka DS, Dunwiddie CT, Persinger CC, Munos BH, Lindborg SR, et al. How to improve R\&D productivity: the pharmaceutical industry's grand challenge. Nat Rev Drug Discov 2010;9:203-214. doi: 10.1038/ nrd3078.

2. Moon $\mathrm{H}$. Clinical Consulting for efficient and successful drug discovery. Jul 2018. https://www.konect.or.kr/Konect_Newsletter/files/12th/article2. pdf

3. Chee D. Personal Communication. 2019 